\title{
EL DERECHO DE RECTIFICACIÓN EN PERIODO ELECTORAL: COMENTARIOS A PROPOSITO DE UN CASO RECIENTE
}

MAR ESQUEMBRE VALDÉS

Área de Derecho Constitucional

El que domina los medios de comunicación de masas, domina al electorado; el que domina al electorado, domina el proceso del poder político.

K. LOEWENSTEIN

\section{INTRODUCCION}

No es necesario poner en evidencia la situación de indefensión en que se encuentra el ciudadano ante el poder de los medios de comunicación social. La libertad de expresión y el derecho a la información de los que cada ciudadano es titular, que son garantía de la existencia de una opinión pública libre -condición necesaria para el recto ejercicio de todos los demás derechos en que se fundamenta el sistema político democrático, como ha proclamado nuestro Tribunal Constitucional en reiteradas ocasiones-, han decaído en favor del discurso mercantil impuesto por los medios, los cuales, obviando los principios a que deben someterse en una sociedad plural y democrática, han abandonado su primigenia función de control al poder clásico para convertirse en otro poder más con una desmesurada influencia pública.

El ciudadano no posee ningún cauce para decidir qué mensajes le interesan, no puede establecer el contenido de la comunicación, casi no influye en su posible alteración y, llegado el momento en que realmente se requiere su participación en los asuntos públicos mediante la elección libre de sus representantes políticos, los medios de comunicación intentan también suplantar su voluntad mediante la creación de estados de opinión favorables a sus pretensiones, dadao que la comunicación directa entre electores y candidatos se ha sustituído por los mensajes lanzados por los propios medios, conformando, gracias a su capacidad de contacto con la población, la opinión púlica. Es obvia, pues, la necesidad de incrementar los cauces de participación del individuo en la conformación de la opinión pública. 
El derecho de rectificación ha pretendido convertirse en uno de estos cauces de participación, en una posibilidad de acceso de los ciudadanos a los canales de comunicación a través de los medios de los que la gran mayoría carece. Pero las condiciones en que el citado derecho ha sido establecido hacen que éste sea escasamente efectivo y ello, unido al poco interés que ha despertado su estudio en la doctrina, lo ha hecho caer, tanto en la praxis como desde la perspectiva teórica, en el olvido. Aunque fugazmente y de forma tangencial parece asomarse tímidamente, como ha ocurrido recientemente con ocasión de una información publicada por un diario nacional en pleno periodo electoral; esta situación se ha presentado como especialmente idónea para reivindicar la presencia en nuestro ordenamiento jurídico del casi inexistente derecho de rectificación. Por ello, el objeto de este trabajo no es ofrecer vías de revisión del derecho de rectificación -que por otro lado lo harían demasiado extenso-, sino que, simplemente, se pretende recordar, al hilo de un caso reciente, en qué consiste este derecho, diferenciándolo de otros mecanismos que pudieran introducir confusiones en su concepción, y constatar la necesidad de un planteamiento del mismo más acorde con la realidad actual a fin de que contribuya a que el al proceso de formación de la opinión pública sea, efectivamente, libre, de acuerdo con los valores superiores de nuestro ordenamiento jurídico.

\section{EL DERECHO DE RECTIFICACION DE LA LEY ORGANICA 2/1984, DE 26 DE MARZO}

El primer párrafo del artículo 1 de la Ley Orgánica 2/84, de 26 de Marzo, reguladora del Derecho de Rectificación (en adelante L.O.D.R.), fija el contenido del citado derecho al establecer que «Toda persona, natural o jurídica, tiene derecho a rectificar la información difundida por cualquier medio de comunicación social, de hechos que le aludan, que considere inexactos y cuya divulgación pueda causarle perjuicio». Sin embargo, no proporciona una definición del citado derecho.

La Real Academia de la Lengua define el término «rectificar» como «contradecir a otro en lo que ha dicho, por considerarlo erróneo» 1 . Sobre esta base, podríamos definir al derecho de rectificación como la posibilidad de que goza cualquier persona, natural o jurídica, aludida en una información difundida por un medio de comunicación social de contradecir la misma por considerarla errónea o inexacta y susceptible de causarle un perjuicio.

La forma y condiciones de ejercicio de este derecho se establecen en los artículos 2 al 4 de la L.O.D.R.. En líneas generales, la forma de ejercicio del derecho consiste en la remisión de un escrito de rectificación al director del medio de comunicación, en un plazo de siete días desde que se publicó o difundió la información que se

1 REAL ACADEMIA ESPAÑOLA: Diccionario de la Lengua Española, 21 ${ }^{\text {a }}$ Edición, Espasa-Calpe, 1994. 
desea rectificar; dicho escrito debe limitarse a los hechos de la información y su extensión no debe superar a la que tuvo ésta. El director del medio de comunicación debe publicar o difundir, de forma íntegra, la rectificación en un plazo de tres días desde que la recibió, sin comentarios ni apostillas y con una relevencia semejante a la que tuvo la información rectificada.

En el caso de que el derecho ejercitado correctamente por su titular sea vulnerado, el rectificante goza de una acción de rectificación prevista en la L.O.D.R. (art.5 y ss.), que se sustancia por el procedimiento de los juicios verbales, con algunas modificaciones 2 .

La finalidad del derecho de rectificación de defensa de los derechos individuales o los intereses legítimos de la persona frente a la información difundida por los medios de comunicación social se ha querido interpretar por algún autor como un límite a la libertad de información del artículo 20.1.d) de la Constitución ${ }^{3}$, añadido al límite específico que el citado precepto establece en su apartado cuarto. No se puede entender así y en este sentido se ha pronunciado el Tribunal Constitucional en los siguientes términos: «...la rectificación (...) no menoscaba el derecho fundamental proclamado por el artículo 20.1.d) (...), ni siquiera en el caso de que la información que haya sido objeto de rectificación pudiera revelarse como cierta y ajustada a la realidad de los hechos. (...) el simple disentimiento por el rectificante de los hechos divulgados no impide al medio de comunicación social afectado difundir libremente la información veraz (...) la simple inserción de una versión de los hechos distinta y contradictoria nio siquiera limita la facultad del medio de ratificarse en la información inicialmente suministrada o, en su caso, aportar y divulgar todos aquellos datos que la confirmen o avalen» 4.

La versión apuntada del derecho de rectificación (= límite a la libertad de información) no es más que el reverso de aquella otra que lo presenta como manifestación de la libertad de información. Así, el derecho de rectificación aparece como concreción del derecho a comunicar información del artículo 20.1.d) de la Constitución, ya que éste pertenece a toda la colectividad y no sólo a los periodistas, como así lo ha afirmado el Tribunal Constitucional en numerosas ocasiones ${ }^{5}$.

2 Los aspectos procesales de la acción de rectificación han sido estudiados por LORCA NAVARRETE, A.: EI nuevo proceso civil de rectificación, Revista La Ley, nº 3, 1984.

3 Así lo sostiene CARRILLO SALCEDO, M.: Libertad de expresión y derecho de rectificación en la C.E. de 1978, en Revista de Derecho Político, nº 23, 1986; el mismo: Derecho a la información y veracidad informativa (comentario a las SSTC 168/86 y 6/88), en Revista Española de Derecho Constitucional, $\mathrm{n}^{\mathrm{o}} 23,1988$.

4 STC 168/86, F.J. 5.

5 Así, la STC 168/86 niega que sólo sean sujetos del derecho a comunicar información «los titulares del órgano o medio difusor de la información o los profesionales del periodismo o quienes, aun sin serlo, comunican una información a través de tales medios, sino, primordialmente, la colectividad y cada uno de sus miembros» (F.J. 2). También en el mismo sentido SSTC 165/87, F.J. 10 y 6/88, F.J. 5. 
Cabría afirmar, siguiendo a LOPEZ ULLA ${ }^{6}$, que el derecho de rectificación, atendiendo a su actual configuración legal, constituye un límite a la libertad de información del rectificante, tanto por su objeto, limitado hasta extremos que lo hacen como a continuación veremos- totalmente inoperante, como por el sujeto, determinado por excesivos requisitos legitimadores, así como por otros aspectos establecidos en la L.O.D.R. a los que no haremos referencia por no extendernos demasiado.

Respecto del objeto de la rectificación, se presenta con claridad en la L.O.D.R., cuyo artículo 1 establece que se podrá rectificar «la información difundida (...) de hechos que le aludan, que considere inexactos», lo cual reitera en el segundo apartado del artículo 2: "La rectificación deberá limitarse a los hechos de la información que se desea rectificar». Así, la Ley restringe el ámbito de aplicación de este derecho a la difusión de hechos considerados inexactos por el rectificante. Ello requiere fijar la atención en dos cuestiones básicas: La veracidad informativa y el concepto de información.

En referencia a la veracidad informativa, se ha considerado por algún sector doctrinal que el bien jurídico protegido por el derecho de rectificación, aunque de forma indirecta, es el derecho constitucional a recibir una información veraz. Pero ello no supone que la información que se difunda haya de ser necesariamente cierta 7 . Tampoco viene a significar $-\mathrm{y}$ el texto de la L.O.D.R. es bien claro al respecto- que el rectificante haya de probar que los hechos no son veraces, sino que basta la apreciación subjetiva de la falsedad o inexactitud de los mismos ${ }^{8}$. De ello se desprende que el derecho de rectificación no es un derecho a hacer prevalecer una información sobre otra, sino algo mucho más simple: un derecho de réplica, es decir, un derecho a ofrecer otra versión, a contradecir una información respecto de la cual se disiente 9.

Así, el derecho de rectificación posibilita que una misma información pueda ser contemplada desde dos perspectivas distintas, derivando de ello la existencia de una

6 LOPEZ ULLA, J.M.: Libertad de informar y derecho a expresarse: la jurisprudencia del Tribunal Constitucional, Servicio de publicaciones de la Universidad de Cádiz, Cádiz, 1994, pg. 134.

7 Así lo ha interpretado el $\mathrm{TC}$ al afirmar que «cuando la Constitución requiere que la información sea "veraz" no está tanto privando de protección a las informaciones que puedan resultar erróneas - o sencillamente no probadas en juicio-cuando estableciendo un especifico deber de diligencia sobre el informador, a quien se le puede y deb exigir que lo que transmita como "hechos" haya sido objeto de previo contraste con datos objetivos. (...) las afirmaciones erróneas son inevitables en un debate libre, de tal forma que, de imponerse la "verdad" como condición para el reconocimienot del derecho, la única garantía de seguridad jurídica sería el silencio» (STC 6/88, F.J. 5). En el mismo sentido, más recientemente, STC 41/94, F.J. 3.

8 En este sentido, el TC ha señalado que «la inserción de la rectificación interesada en la publicación o medio de difusión no implica la exactitud de su contenido, pues ni siquiera la decisión judicial que ordene dicha inserción puede acreditar, por la propia naturaleza del derecho ejercitado y los límites en que se desenvuelve la acción de rectificación, la veracidad de aquélla» (STC 168/86, F.J. 5).

9 Así lo considera SAIZ DE MARCO, I.: Sobre el derecho de rectificación, en Revista La Ley, $\mathrm{n}^{\mathbf{0}} 1,1992$, pg. 877. 
discusión libre entre las partes que redunda en una mejor formación de la opinión pública ${ }^{10}$. Pero debido a la preponderancia de los medios -que son los habituales sujetos emisores- la verosimilitud de los hechos por ellos divulgados se convierte, para la colectividad receptora, en veracidad. Y a ello cabría añadir que, en cualquier caso, el medio de comunicación social siempre tiene la posibilidad de difundir, al mismo tiempo, cualquier comentario relativo a la rectificación efectuada, lo cual coloca al rectificante en situación de desigualdad, al no poder éste replicar o contraargumentar dicho comentario.

Respecto del concepto de información, hay que señalar, en primer lugar, que el reconocimiento progresivo por la jurisprudencia constitucional de la existencia de dos derechos autónomos en el artículo 20.1 de la Constitución ${ }^{11}$ ha tenido como punto de partida y argumento fundamental la delimitación del objeto que a cada uno se le atribuye. Así, mientras que el objeto de la libertad de expresión son las opiniones, creencias y juicios de valor, el objeto de la libertad de información se limita a los hechos.

En esta línea, el Tribunal Constitucional ha considerado que el objeto del derecho a la información está constituído por el «conjunto de hechos que pueden considerarse como "noticiables" o "noticiosos", entendiendo por tales aquéllos que puedan encerrar trascendencia pública y que sean necesarios para que la participación de los ciudadanos en la vida colectiva sea real» (STC 105/83, F.J. 11). Sin embargo, también ha entendido el Alto Tribunal que la narración de hechos no se puede dar asépticamente, de tal forma que únicamente se lleve a cabo la presentación pura de hechos ${ }^{12}$. Admitiéndose, sobre esta base, que hechos no se puede entender restrictivamente, siendo de aplicación a los medios como emisores habituales y que el conocimiento de esos hechos es necesario para la efectiva participación de la colectividad en los asuntos públicos, nada debiera objetarse respecto de la aplicación del mismo tratamiento a los particulares que hacen uso del derecho de rectificación. Ello no obstante, el Tribunal Constitucional ha interpretado restrictivamente el concepto de hechos cuando se trata del sujeto pasivo de la relación informativa, privilegiando, de esta forma, al habitual emisor o sujeto activo, esto es, a los medios de comunicación social.

10 Vide LOPEZ ULLA, J.M.: Libertad de informar.., op. cit., pg. 131. También BOUZAT, G.: Libertad de expresión y estructura social: el derecho de réplica, en Revista del Centro de Estudios Constitucionales, $\mathrm{n}^{\circ} 3,1989$, pg. 99.

11 SSTC 105/83, F.J. 11; 168/86, F.J. 2; 6/88, F.J. 5; 51/89, F.J. 2; 105/90, F.J.4; 214/91, F.J. 6; 223/92, F.J. 1; 232/93, F.J. 1; 31/94, F.J. 7; 41/94, FF. JJ. 3 y 4; 320/94, F.J. 3.

12 En este sentido se ha pronunciado la STC 171/90, F.J. 10 y, más recientemente, el ATC de 27 de Marzo de 1995, que dispone que «el derecho a la información no impone al peridista la publicación de noticias escuetas y rigurosamente asépticas en función de los hechos o acaecimientos noticiables» (F.J. 6). 


\section{ESPECIALIDADES DEL DERECHO DE RECTIFICACION. EL DERECHO DE RECTIFICACION EN PERIODO ELECTORAL Y LOS MECANISMOS ADMINISTRATIVOS DE RECTIFICACION}

Los escasos estudios existentes sobre el derecho de rectificación se limitan al analisis de la L.O.D.R., pero en ellos se observa la ausencia de toda referencia a las modalidades especiales que del mismo existen 13. Dichas especialidades podrímos reducirlas sólo a una en concreto, la establecida en la Ley Orgánica 5/1985, de 19 de junio, del Régimen Electoral General (LOREG en adelante) ${ }^{14}$.

El artículo 68 de la LOREG contempla el derecho de rectificación en los siguientes términos:

«Cuando por cualquier medio de comunicación social se difundan hechos que aludan a candidatos o dirigentes de los partidos, federaciones, coaliciones o agrupaciones que concurran a la elección, que éstos consideren inexactos y cuya divulgación pueda causarles perjuicio, podrán ejercitar el derecho de rectificación de conformidad con lo establecido en la Ley Orgánica 2/1984, de 23 de marzo, con las siguientes especialidades:

a) Si la información que se pretende rectificar se hubieradifundido en una publicación cuya periodicidad no permita divulgar la rectificación en los tres días siguientes a su recepción, el director del medio de comunicación deberá hacerla publicar a su costa dentro del plazo indicado en otro medio de la misma zona y de similar difusión.

b) El juicio verbal regulado en el párrafo segundo del artículo 5 de la mencionada Ley Orgánica se celebrará dentro de los cuatro días siguientes al de la petición».

El derecho de rectificación contemplado en la LOREG es igual al establecido en la L.O.D.R., como indica expresamente el precepto citado, si bien con las especialidades apuntadas, consistentes en, por un lado, una limitación de los plazos de tiempo, medida del todo lógica si tenemos en cuenta que el citado derecho está contem-

13 Sólo SAIZ DE MARCO, I.: Sobre el derecho..., op. cit., pg. 876, alude brevemente a las mismas.

14 Respecto de las especialidades restantes, se trata de los preceptos reguladores del derecho de rectificación establecidos en las leyes creadoras de algunas entidades autónomas de radiotelevisión. Dado que dichas leyes fueron promulgadas con anterioridad a la L.O.D.R., deben entenderse tácitamente derogadas por ésta última, ya que el régimen contemplado en las mismas para el ejercicio del citado derecho era similar al establecido en el Estatuto de Radiotelevisión Española, precepto expresamente derogado por la Disposición Derogatoria de la L.O.D.R. 
plado para su ejercicio en periodo electoral y la duración de éste es sumamente limitada, y, por otro, en la reducción de la fórmula legitimadora respecto a la empleada en la L.O.D.R., ya que mientras que esta última hace referencia a «Toda persona, natural o jurídica», la LOREG alude sólo a «candidatos o dirigentes de los partidos, federaciones, coaliciones o agrupaciones que concurran a la elección».

Sin embargo, en la LOREG nos encontramos, en su artículo 69, otro supuesto de rectificación que no parece encuadrable en los supuestos anteriores. A propósito de las encuestas electorales, el citado artículo establece, en su apartado cuarto, lo siguiente:

"Los medios informativos que hayan publicado o difundido un sondeo, violando las disposiciones de la presente Ley, están obligados a publicar y difundir, en el plazo de tres días las rectificaciones requeridas por la Junta Electoral Central, anunciando su procedencia y el motivo de la rectificación, y programándose o publicándose en los mismos espacios o páginas que la información rectificada».

En el apartado siguiente del mismo precepto legal se prevé la posibilidad de plantear, frente a las resoluciones de la Junta Electoral Central (en adelante JEC), recurso ante la jurisdicción contencioso administrativa.

En relación a lo ya expuesto sobre el derecho de rectificación, dos notas llaman inmediatamente la atención en esta, pudiéramos llamarla así, rectificación especial. En primer lugar, el sujeto activo de la rectificación, que es exclusivamente la JEC, esto es, un órgano de la Administración Electoral, concretando así la legitimación activa, que en los casos anteriores presentaba una pluralidad; y, en segundo lugar, el carácter marcadamente imperativo de la fórmula utilizada en el precepto al referirse a la obligación de publicación y difusión de la rectificación por parte del medio de comunicación social, puesto que mientras que en la L.O.D.R. -de aplicación también para el supuesto del artículo 68 de la LOREG- se establece que «el director del medio de comunicación social deberá publicar o difundir...», la LOREG dispone que «Los medios (...) están obligados a publicar y difundir...».

Ambas notas evocan la regulación dual que del -al parecer- actualmente unificado derecho de rectificación existía en el dictatorial régimen anterior, puesto que la Ley de Prensa e Imprenta de 1966, parcialmente vigente en la actualidad, distinguía entre derecho de rectificación y derecho de réplica en base al criterio del sujeto activo titular del derecho. El artículo 58 de la citada Ley, desarrollado por el Decreto regulador 746/1966, de 31 de marzo, establecía, en su primer apartado, el derecho de réplica para «Toda persona natural o jurídica, que se considere injustamente perjudicada por cualquier información escrita o gráfica que la mencione o aluda, inserta en una publicación periódica». Y el artículo 62 de la misma Ley, desarrollado por Decreto regulador 745/1966, de 31 de marzo, instituía el derecho de rectificación para la Administración Pública, disponiendo que «Los Directores de las publicacio- 
nes periódicas están obligados a insertar gratuitamente (...) cuantas notas o comunicados les remitan la Administración o Autoridades (...), rectificando o aclarando información publicada en aquélla sobre actos propios de su competencia o función».

Así, podemos observar un reflejo del derecho de rectificación contemplado en la Ley de 1966 en lo preceptuado en los apartados 4 y 5 del artículo 69 de la LOREG, ya que en ambos casos concurren tres circunstancias similares: en primer lugar, la naturaleza pública del sujeto activo del derecho, ya que en ambos casos se trata de órganos de la Administración, si bien el la LOREG únicamente está legitimada la JEC; en segundo lugar, la utilización de la misma formula imperativa (Están obligados) en lo que respecta a la publicación y difusión de la rectificación por parte de los medios de comunicación, aunque el Decreto regulador del derecho de rectificacion de la Ley de 1966 fijaba la imposición de una sanción administrativa para el caso de incumplimiento de dicha obligación por parte del medio, mientras que la LOREG no prevé ningún tipo de sanción; y, en tercer lugar, se contempla en ambas la posibilidad de que el sujeto pasivo pueda recurrir la decisión de la Administración en la vía contencioso administrativa.

Aunque el espacio temporal de vigencia de las leyes citadas sea completamente diferente, ya que el anterior régimen si por algo se caracterizaba era por una carencia material de libertades, no podemos evitar el pensamiento fugaz de encontrarnos en este caso ante una reminiscencia de la antigua censura. Desechada esta idea, podemos sostener que el supuesto del artículo 69 de la LOREG no es una especialidad del derecho de rectificación regulado por la L.O.D.R., sino que se trata de un mecanismo de que goza la Administración Electoral para cumplir con la finalidad general de garantizar «la transparencia y objetividad del proceso electoral y del principio de igualdad» 15 , esencial para la protección del proceso de libre formación de la opinión pública.

\section{RECTIFICACION EN PERIODO ELECTORAL: UN CASO RECIENTE}

El ejercicio del derecho de rectificación de la L.O.D.R. -así como su especialidad del artículo 68 de la LOREG- no es muy frecuente ${ }^{16}$, y tampoco lo es la rectificación prevista en el artículo 69 de la LOREG. Sin embargo, con ocasión de las pasa-

15 artículo 8.1 LOREG.

16 Aunque se suele hacer uso de este derecho, su forma y condiciones de ejercicio resultan ser en la praxis distintas a las establecidas legalmente, ya que las rectificaciones en la mayoría de ocasiones, se insertan como «aclaraciones» en el apartado de «Cartas al Director» o bien constan, en un pequeño recuadro marginal que nunca se extiende más allá de los treinta caracteres, como «Fe de Errores». Lo dicho es aplicable a la prensa, que aún ofrece esa ínfima posibilidad, en los restantes medios de comunicación es algo prácticamente desconocido. 
das elecciones generales se produjo un incidente que catapultó a la actualidad, aunque sólo efímeramente, si no ambos supuestos, sí el mencionado en último lugar.

El pasado 14 de febrero, esto es, dentro del periodo electoral, el diario $\mathrm{ABC}$ publicó una información sobre una macroencuesta de intención de voto realizada por el Centro de Investigaciones Sociológicas (CIS) a instancias del Gobierno -dicha información ocupaba una extensión de dos páginas en un cuadernillo central dedicado a las elecciones generales- ${ }^{17}$. Ese mismo día, D. Joaquin Arango, Presidente del CIS, realiza unas declaraciones en un medio radiofónico -concretamente, la Cadena SER-, manifestando que la información publicada por el citado diario es falsa puesto que el CIS no ha realizado ninguna encuesta. También en el curso del mismo día, el representante de Izquierda Unida ante la JEC solicita a este órgano que estudie la posible vulneración, cometida por $\mathrm{ABC}$, del régimen jurídico de las encuestas electorales recogido en el artículo 69 de la LOREG, puesto que no se incluía, como es obligatorio, la ficha técnica de la encuesta. Ante esta denuncia, la JEC acuerda otorgar un plazo común de cuatro días al CIS y al Diario $\mathrm{ABC}$ para el traslado de un informe y sus alegaciones, respectivamente.

En el transcurso del plazo citado, el Diario $\mathrm{ABC}$ publica el texto íntegro de un comunicado remitido por el CIS rectificando la información sobre la macroencuesta, en el sentido de desmentir que dicho organismo haya realizado ningún tipo de encuesta ${ }^{18}$, y seguidamente, inserta una nota de su redacción, en destacados caracteres tipográficos respecto del texto de rectificación del CIS, en la que formula distintos tipos de acusaciones contra este organismo, susceptibles de perjudicar su prestigio ${ }^{19}$. Posteriormente, todavía dentro del plazo para la presentación de sus alegaciones ante la JEC, ABC publica una extensa información sobre el Presidente del CIS, lanzando duros ataques en un intento de demostrar su falta de fiabilidad ${ }^{20}$.

En su escrito de alegaciones ante la JEC, ABC solicitó que se requiriera a Izquierda Unida y al Partido Popular a efectos de que ambos certificaran si habian tenido o no conocimiento de los trabajos de campo realizados por el CIS, a lo que contesta en sentido afirmativo el Partido Popular, mientras que Izquierda Unida certifica desconocer la existencia de dichos trabajos. Esta circunstancia se publica por $\mathrm{ABC}$ el 23 de febrero, junto con otras informaciones relativas, todas ellas, al poco

17 Vide Diario ABC del 14/02/96, pg. 22 y 23.

18 Vide Diario ABC del 16/02/96, pg. 24.

19 Dichas acusaciones atentarían, probablemente, si de una persona física se tratase, contra el honor de la misma, del que no cabe hablar respecto de las personas jurídicas, pudiendo ser éste sustituído, en este caso, por la fama, prestigio o buen nombre, como ha reiterado, en numerosas ocasiones, el Tribunal Constitucional.

20 Vide Diario ABC del 18/02/96, pg. 28 y 29. El titular, en grandes caracteres tipográficos, ya es bastante ilustrativo: «El Presidente del CIS también mintió en las elecciones del 28-M». 
crédito que merece el organismo ya mencionado y que colocan al Diario como único defensor de la verdad ${ }^{21}$. Sin embargo, ese mismo día, la JEC instó al citado Diario a que publicase, en un plazo de tres días, un texto de rectificación sobre la encuesta atribuida al CIS, en el sentido de que la información aparecida en ese Diario el 14 de febrero no podía ser presentada como una encuesta en los términos del artículo 69 de la LOREG, debiendo figurar dicho texto de rectificación en la portada y en las páginas interiores correspondientes. Transcurrido dicho plazo, la JEC acordó, el 27 de febrero, reiterar a $\mathrm{ABC}$ que debía rectificar «de inmediato» dicha información. El Diario $\mathrm{ABC}$ comunicó a la JEC la interposición de un recurso contencioso administrativo contra esta resolución, alegando que la misma era gravemente perjudicial para el periódico, al dejar en entredicho su fiabilidad y afectar a la libertad de expresión. Presumimos que el asunto está siendo resuelto ante la jurisdicción contencioso administrativa, puesto la rectificación impuesta por la JEC no ha sido publicada, hasta la fecha, por ABC.

La dinámica seguida en el caso referido es un claro e indiscutible ejemplo de aplicación del mecanismo administrativo de rectificación contemplado en la LOREG, sin embargo, a la luz de lo ocurrido, se revelaba también aplicable el derecho de rectificación regulado en la L.O.D.R., estando legitimado activamente el Presidente del CIS desde una doble perspectiva: como persona natural, ya que se publicó una información que le aludía directamente y era susceptible de causarle un perjuicio, y, por otra parte, en calidad de representante legal de una persona jurídica, puesto que las informaciones publicadas ponían en entredicho el prestigio del CIS. Ambos tipos de rectificación -rectificación como mecanismo administrativo y como derecho subjetivo-, son perfectamente compatibles, sin que el ejercicio de uno de ellos impida el del otro; sin embargo, el Presidente del CIS optó por el ortodoxo método de acudir a un medio de comunicación distinto para dar su versión de los hechos. Este hecho, unido al infructuoso intento de la Administración Electoral de rectificar una información que pudiera revelarse como falsa, que, en cualquier caso, empañó la transparencia que debe presidir todo proceso electoral, y a la escasísima notoriedad que el caso relatado alcanzó en los medios de comunicación social, constituye un gráfico ejemplo de la ineficacia, tanto del derecho de rectificación, como del mecanismo -que no derecho- rectificador de la Administración, potenciada por el interés de los medios de comunicación social en desterrar por completo, e incluso suprimir, cualquier atisbo de existencia de un cauce de participación en los mismos de otros sectores ajenos a ellos, en aras de enriquecer el debate de los asuntos públicos.

Así, pues, queda patente la ineficacia, tanto de la rectificación administrativa, como del derecho de rectificación regulado en la L.O.D.R., para contribuir al proce-

21 Vide Diario ABC del 23/02/96, pg. 27 y 28. También en este caso el titular es significativo: «El PP golpea duramente a Arango al comunicar a la Junta Electoral que conocía la encuesta del CIS»: 
so de libre formación de la opinión pública, sobre todo en los periodos en los que se agudiza el papel de influencia de la misma en la representación política, y ello por la inexistencia, por parte del poder legislativo, que bien podría obedecer a la presión de los grandes grupos económicos detentadores de los medios de comunicación, de una voluntad de revisión y actualización de los cauces de participación de los ciudadanos en el proceso comunicativo. 